\title{
Usefulness of Repeat Angiography to Establish Spontaneous Coronary Artery Dissection in Small Vessels
}

\author{
Naoki Kubota, MD; Kazuyuki Ozaki, MD, PhD; Yasuhiko Tanabe, MD, PhD; \\ Yasuhiro Iwasaki, MD; Tsuyoshi Yoshida, MD, PhD; Takeshi Okubo, MD; \\ Shinpei Kimura, MD, PhD; Makoto Hoyano, MD, PhD; Takao Yanagawa, MD, PhD; \\ Takeshi Kashimura, MD, PhD; Tohru Minamino, MD, PhD
}

\begin{abstract}
Background: Spontaneous coronary artery dissection (SCAD) is a rare disease that is often misdiagnosed, except in typical cases. Although intracoronary imaging and multislice coronary computed tomography angiography (CCTA) are useful in establishing dissection, they may not be feasible in all instances, especially in small vessels.

Methods and Results: We describe a series of 7 patients with acute coronary syndrome secondary to small vessel SCAD that was detected only upon repeat coronary angiography (CAG). This cohort had a mean ( $\pm S D$ ) age of $50 \pm 6$ years, was predominantly female $(n=6 ; 86 \%)$, and had few coronary risk factors. Three patients $(43 \%)$ had dissection of the distal segment of the right coronary artery, $3(43 \%)$ had distal left circumflex artery dissection, and 1 patient (14\%) had a diagonal branch dissection. None of the patients required percutaneous coronary intervention, and received conservative therapy only, because the infarct area was sufficiently small. No definitive diagnosis of SCAD could be established in any of the patients at first admission because CAG alone or CCTA did not reveal the presence of a flap or intraluminal hemorrhage. However, in such patients without a definitive diagnosis, repeat CAG in the chronic stage showed enlargement of vessels, suggesting the healing of an SCAD.
\end{abstract}

Conclusions: Repeat CAG may be useful for suggesting the occurrence of SCAD.

Key Words: Coronary angiography; Diagnosis; Spontaneous coronary artery dissection

$\mathbf{S}$ pontaneous coronary artery dissection (SCAD) is defined as the dissection of an epicardial coronary artery that is not associated with atherosclerosis or trauma and is not iatrogenic. SCAD is a rare disease, and its reported prevalence in acute coronary syndrome (ACS) ranges between $0.1 \%$ and $4 \% .^{1,2} \mathrm{ACS}$ is defined as unstable angina and acute myocardial infarction. ${ }^{3}$ Coronary angiography (CAG) is one of the major diagnostic modalities for SCAD. The Saw coronary angiogram classification for $\mathrm{SCAD}^{4}$ defines 3 types of CAG findings: Type 1, which refers to the pathognomonic angiographic appearance of SCAD with contrast dye staining of the arterial wall and the presence of multiple radiolucent lumens; Type 2, which refers to the presence of diffuse stenosis of varying severity and length; and Type 3, which represents focal or tubular stenosis, usually $<20 \mathrm{~mm}$ in length, that mimics atherosclerosis. Although Type 1 SCAD is clearly recognized as a flap during CAG, Types 2 and 3 are sometimes impossible to diagnose by CAG alone, and intracoronary imaging techniques such as intravascular ultrasonography (IVUS) or optical coherence tomography (OCT), as well as multislice coronary computed tomography angiography (CCTA), are useful for establishing SCAD. Nonetheless, diagnosing SCAD in small vessels is challenging because of technical issues, such as non-delivery of the intracoronary imaging device or insufficient resolution for detection using CCTA.

Here we describe a series of 7 cases in which SCAD in small vessels was detected only on repeat CAG in the chronic stage.

\section{Patient Characteristics}

Between 2010 and 2019, 7 patients with SCAD in the small vessels were treated at Niigata University Medical and Dental Hospital and Niigata Prefectural Shibata Hospital.

Received September 10, 2020; accepted September 10, 2020; J-STAGE Advance Publication released online October 29, 2020 Time for primary review: 1 day

Department of Cardiovascular Biology and Medicine, Niigata University Graduate School of Medical and Dental Sciences, Niigata (N.K., K.O., T.O., S.K., M.H., T. Yanagawa, T.K., T.M.); Department of Cardiology, Niigata Prefectural Shibata Hospital, Niigata (Y.T., Y.I., T. Yoshida), Japan

Mailing address: Naoki Kubota, MD, Department of Cardiovascular Biology and Medicine, Niigata University Graduate School of Medical and Dental Sciences, 1-757 Asahimachidori, Chuo-ku, Niigata 951-8510, Japan. E-mail: naokikub@med. niigata-u.ac.jp

All rights are reserved to the Japanese Circulation Society. For permissions, please e-mail: cr@j-circ.or.jp

ISSN-2434-0790 


\begin{tabular}{|c|c|c|c|c|c|c|c|c|c|c|c|}
\hline \multirow{2}{*}{$\begin{array}{l}\text { Patient } \\
\text { no. }\end{array}$} & \multirow{2}{*}{ Sex } & \multirow{2}{*}{$\begin{array}{c}\text { Age } \\
\text { (years) }\end{array}$} & \multirow{2}{*}{$\begin{array}{l}\text { Presentation at } \\
\text { admission }\end{array}$} & \multicolumn{4}{|c|}{ Coronary risk factors } & \multirow{2}{*}{$\begin{array}{l}\text { Dissection } \\
\text { site }\end{array}$} & \multirow{2}{*}{ TIMI flow } & \multirow{2}{*}{ Therapy } & \multirow{2}{*}{$\begin{array}{c}\text { Peak CK } \\
\text { (IU/L) }\end{array}$} \\
\hline & & & & Diabetes & HT & HL & Smoking & & & & \\
\hline 1 & Female & 41 & NSTEMI & 0 & 0 & 0 & 0 & 12 & 3 & Conservative & 1,070 \\
\hline 2 & Female & 49 & STEMI & 0 & 0 & 0 & 0 & 12 & 3 & Conservative & 535 \\
\hline 3 & Female & 55 & NSTEMI & 0 & 0 & 1 & 0 & 4PD & 3 & Conservative & 381 \\
\hline 4 & Female & 57 & STEMI & 0 & 0 & 1 & 0 & $4 \mathrm{AV}$ & 3 & Conservative & 287 \\
\hline 5 & Male & 54 & STEMI & 0 & 0 & 0 & 0 & $4 \mathrm{AV}$ & 2 & Conservative & 180 \\
\hline 6 & Female & 43 & NSTEMI & 0 & 0 & 0 & 1 & 9 & 3 & Conservative & 1,523 \\
\hline 7 & Female & 54 & NSTEMI & 0 & 0 & 0 & 0 & 12 & 3 & Conservative & 135 \\
\hline
\end{tabular}

4AV, atrioventricular branch; 4PD, posterior descending artery; CK, creatine kinase; DM, diabetes; HL, hyperlipidemia; HT, hypertension; NSTEMI, non-ST-elevation myocardial infarction; STEMI, ST-elevation myocardial infarction; TIMI, Thrombolysis in Myocardial Infarction.
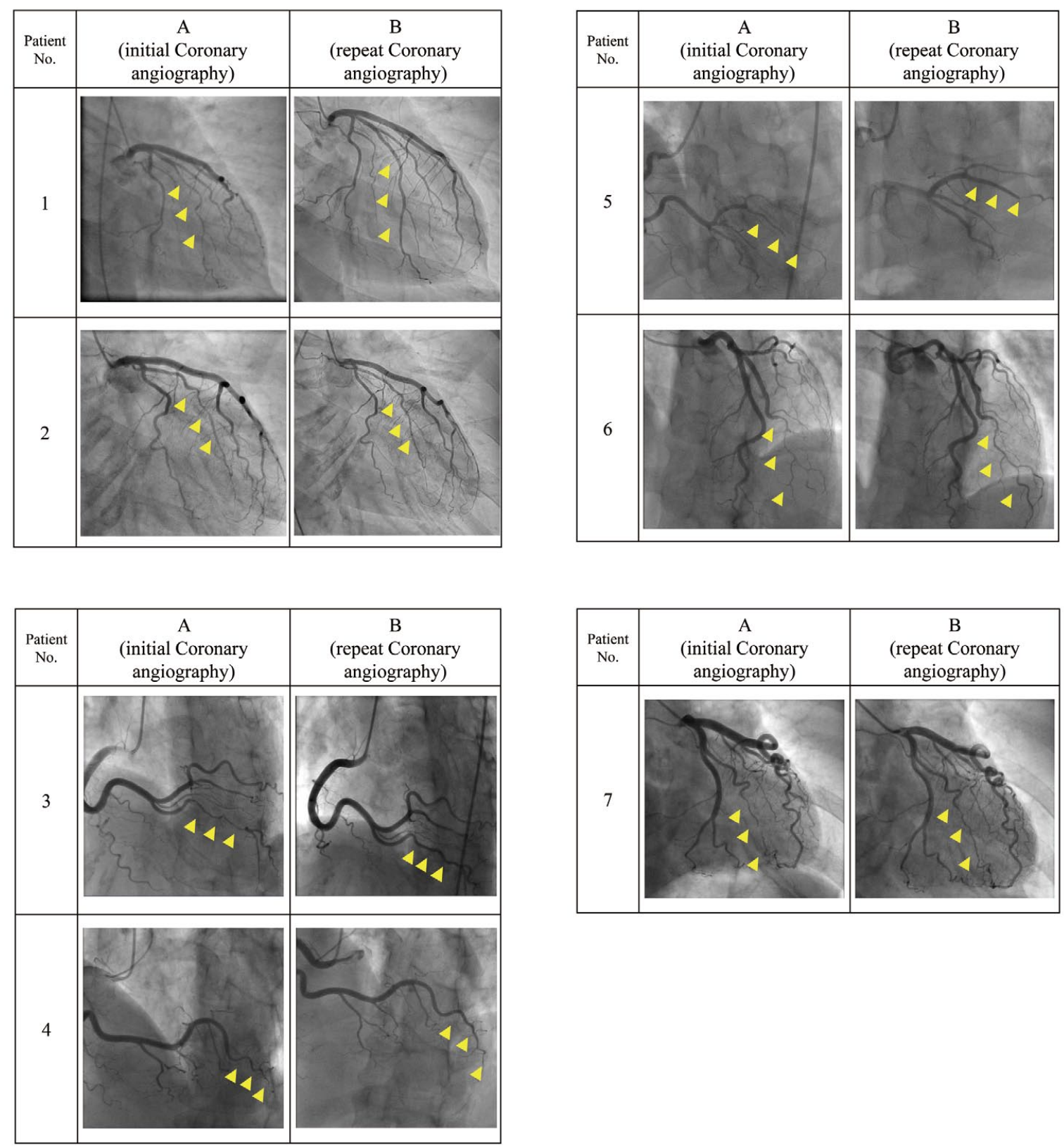

Figure 1. (A) Initial and (B) repeat coronary angiographies in all 7 patients. Arrowheads indicate the location of the dissection. 


\section{A}

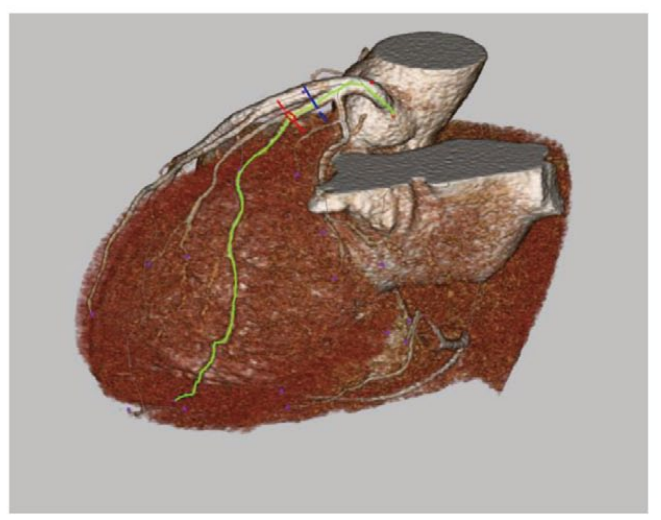

B

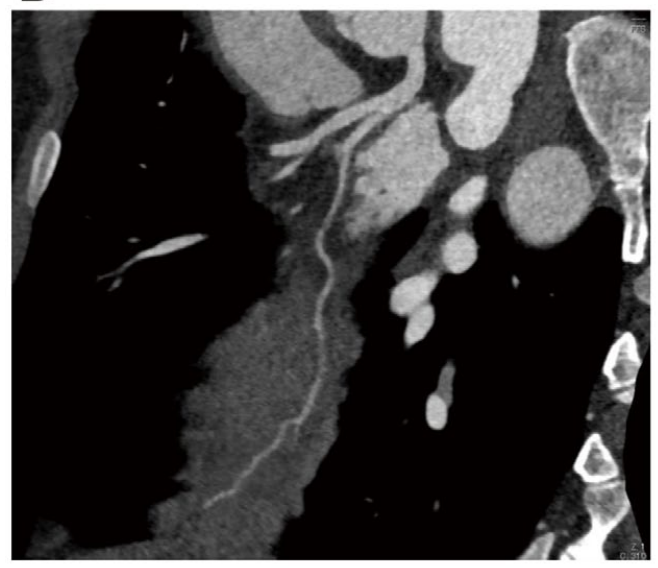

Figure 2. Multislice coronary computed tomography angiography of Patient no. 1. (A) Volume rendering image. (B) Curved multiplanar reformation of the circumflex artery.
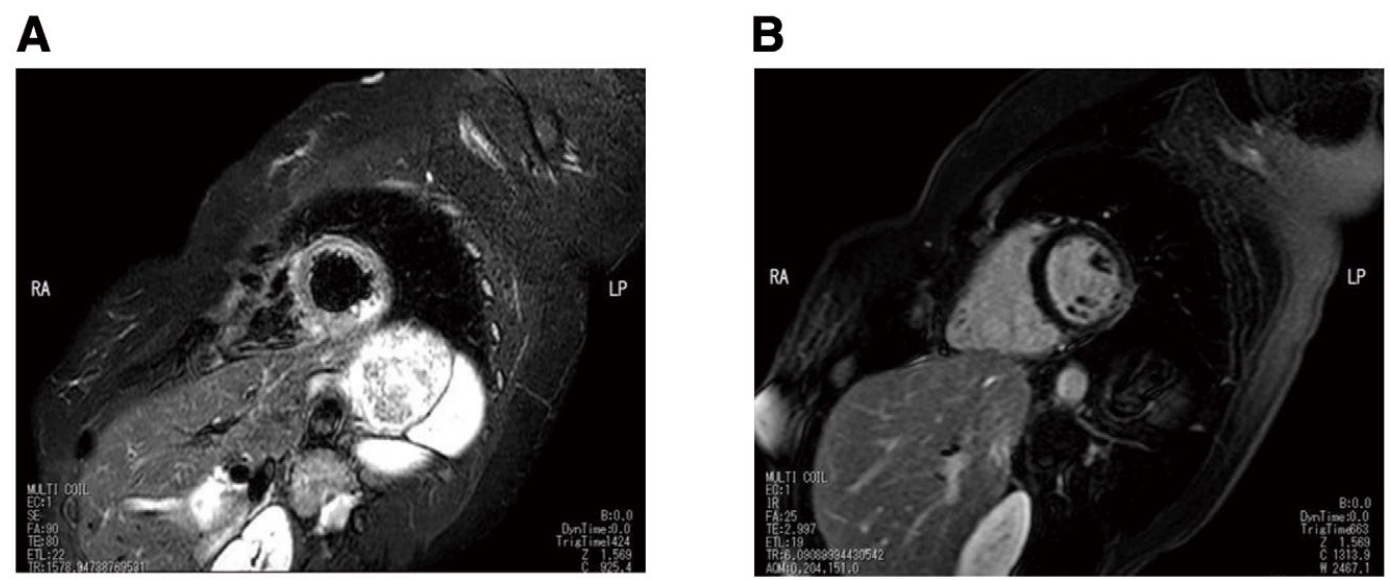

Figure 3. Magnetic resonance imaging of Patient no. 1 showing (A) a T2-weighted image and (B) a late gadolinium-enhanced image.

The baseline characteristics of these patients are given in Table. The mean $( \pm \mathrm{SD})$ patient age was $50 \pm 6$ years, 6 $(86 \%)$ were female, and they had few coronary risk factors. ST-elevation myocardial infarction (STEMI) and nonSTEMI (NSTEMI) at admission were seen in $3(43 \%)$ and $4(57 \%)$ patients, respectively, and none of the patients exhibited cardiogenic shock. The location of the dissection was the distal segment of the right coronary artery (RCA) in 3 patients $(43 \%)$, the distal segment of the left circumflex artery (LCX) in 3 patients (43\%), and the diagonal branch in 1 patient (14\%). None of the patients required percutaneous coronary intervention and all were managed using conservative therapy because the infarct area was small.

Median peak creatine kinase (CK) levels were $381 \mathrm{U} / \mathrm{L}$ (interquartile range [IQR] 234-803 U/L), reflecting the presence of only a small infarction. The patients were dis- charged without any complications. All patients underwent repeat CAG a median of 4 months (IQR 0.75-8 months) after the initial admission. Written informed consent was obtained from all patients before repeat CAG.

Initial and repeat CAGs of all patients are shown in Figure 1. No definitive diagnosis of SCAD could be established at the time of initial admission because neither CAG alone nor CCTA showed the presence of a flap or intraluminal hemorrhage. Nonetheless, in these patients, repeat CAG in the chronic stage showed enlargement of vessels, suggesting healing after SCAD.

We describe in detail the case of 1 typical patient in this cohort (Patient no. 1), in whom a definitive diagnosis of SCAD could not be made at the time of initial admission. Patient no. 1, a 41-year-old woman, presented to the emergency room at Niigata University Medical and Dental 
Hospital with a 1-h history of chest pain. The electrocardiogram showed no significant ST-T change, and transthoracic echocardiography showed no apparent asynergy. The next day, the patients $\mathrm{CK}$ and CK-MB concentrations increased to 1,070 and $101 \mathrm{U} / \mathrm{L}$, respectively, but CCTA using 64-slice multidetector computed tomography (Figure 2) revealed no significant stenosis. However, because $\mathrm{T}_{2}$-weighted and late gadolinium-enhanced magnetic resonance imaging (MRI; Figure 3) showed endocardial infarction in the distal segment of the LCX, cardiac catheterization was performed. CAG revealed diffuse narrowing of the distal segment of the LCX (Figure 1A, Patient no. 1), but with sufficient flow, and vasospasm was not provoked by acetylcholine administration ${ }^{5}$ (i.e., the vasospasm test was negative). Importantly, the narrowing of the LCX was not enlarged by the administration of intracoronary isosorbide dinitrate and, based on MRI findings, this distal segment of the LCX was determined to be the etiological agent of the myocardial infarction. Although the LCX lesion was suspected to be a SCAD, it was impossible to definitely establish the same because pertinent imaging devices could not be delivered. Repeat CAG was performed 9 months later, showing that the LCX lesion had healed and enlarged (Figure 1B, Patient no. 1). These repeat CAG findings are suggestive of the occurrence of SCAD in the distal segment of the LCX at the time of initial presentation.

\section{Discussion}

SCAD is a rare disease, with a reported prevalence in ACS of $0.1-4 \%{ }^{1,2}$ Female sex, fibromuscular dysplasia, vascular tortuosity, a peripartum state, physical and emotional stress, and connective tissue disorders are factors known to be related to SCAD.$^{68}$ The major cause of myocardial injury in SCAD is insufficient coronary flow due to compression by an intramural hematoma or a false lumen. The most commonly involved vessel is the left anterior descending artery and its branches (45-61\% of cases), followed by the LCX and its branches (15-45\%), the RCA and its branches (10-39\%), and the left main trunk in up to $4 \%$ of cases. ${ }^{7,-11}$ In most cases, the mid to distal segments of the coronary arteries are affected, with proximal site involvement seen in only $8.3 \%$ of cases. ${ }^{7}$ According to the Saw coronary angiogram classification system, ${ }^{4}$ SCAD can be classified into 3 types. Type 2 (diffuse smooth stenosis) is the most common angiographic manifestation of SCAD, occurring in up to $67.5 \%$ of cases. This is followed by Type 1 (evident arterial wall stain) in $29.1 \%$ of cases and Type 3 (mimic atherosclerosis) in 3.4\% of cases. ${ }^{7}$

SCAD Types 2 and 3 can be impossible to diagnose by CAG alone. Intracoronary imaging devices such as IVUS and OCT are useful for diagnosing SCAD because they can detect intimal tears, the presence of a false lumen, and intramural hematomas. However, in cases of distal lesions or SCAD in small vessels, intracoronary imaging devices may not be delivered to the lesion site or they may exacerbate SCAD. CCTA is also a useful and non-invasive diagnostic tool; however, small artery dissections (especially those $<2.5 \mathrm{~mm}$ in diameter) may not be detected due to limitations in CT resolution. ${ }^{\mathbf{1 2 - 1 4}}$

In this report we present details of 7 patients with SCAD in the small vessels, which were evident only upon repeat CAG. Saw proposed a stepwise algorithm to diagnose non-atherosclerotic SCAD,${ }^{4}$ according to which early inva- sive CAG is recommended if SCAD is suspected. OCT or IVUS is considered useful for arriving at a definitive diagnosis and, if there are concerns about compromised arterial flow on intracoronary imaging, a repeat angiogram should be performed. However, few studies have reported initial angiogram images and those after healing.

Previous studies have reported that SCAD lesions heal in most patients (70-97\%) without any intervention, but that this could take anywhere between weeks and months. ${ }^{4,9,10,15,16}$ Therefore, conservative therapy is currently recommended for SCAD and percutaneous coronary intervention is not indicated, except if there is evidence of ongoing ischemia, shock, or left main dissection. ${ }^{717}$ Further, if SCAD cannot be established beyond doubt at initial CAG or with other diagnostic techniques, repeat CAG may reveal the healing of the vessels, which suggests initial SCAD occurrence.

A limitation of this study is that it was not possible to prove whether SCAD actually occurred, even if the artery appeared to be healed upon repeat angiography, because the latter does not prove the existence of a dissection. Nonetheless, in cases where SCAD actually occurs in the small vessels but cannot be established at initial admission, repeat angiography to confirm artery healing is useful, because it is suggestive of SCAD.

\section{Conclusions}

In cases where SCAD is suspected but cannot be established at initial CAG or by other diagnostic techniques, repeat CAG may reveal vessel healing, which is suggestive of SCAD occurrence.

\section{Sources of Funding}

This study did not receive any specific funding.

\section{Disclosures}

T.M. is a member of Circulation Journal Editorial Team. The other authors have no conflicts of interest to declare.

\section{IRB Information}

The local ethics committee of the Niigata University School of Medical and Dental Sciences determined that ethics approval for this report was unnecessary.

\section{References}

1. Mortensen KH, Thuesen L, Kristensen IB, Christiansen EH. Spontaneous coronary artery dissection: A Western Denmark Heart Registry study. Catheter Cardiovasc Interv 2009; 74: $710-717$.

2. Nishiguchi T, Tanaka A, Ozaki Y, Taruya A, Fukuda S, Taguchi $\mathrm{H}$, et al. Prevalence of spontaneous coronary artery dissection in patients with acute coronary syndrome. Eur Heart J Acute Cardiovasc Care 2016; 5: 263-270.

3. Sawano M, Yamaji K, Kohsaka S, Inohara T, Numasawa Y, Ando $\mathrm{H}$, et al. Contemporary use and trends in percutaneous coronary intervention in Japan: An outline of the J-PCI registry. Cardiovasc Interv Ther 2020; 35: 218-226.

4. Saw J. Coronary angiogram classification of spontaneous coronary artery dissection. Catheter Cardiovasc Interv 2014; 84: $1115-1122$.

5. Joint Working JCS Group. Guidelines for diagnosis and treatment of patients with vasospastic angina (coronary spastic angina) (JCS 2013): Digest version. Circ J 2014; 78: 2779-2801.

6. Tweet MS, Hayes SN, Pitta SR, Simari RD, Lerman A, Lennon $\mathrm{RJ}$, et al. Clinical features, management, and prognosis of spontaneous coronary artery dissection. Circulation 2012; 31: $579-588$. 
7. Saw J, Aymong E, Sedlak T, Buller CE, Starovoytov A, Ricci D, et al. Spontaneous coronary artery dissection: Association with predisposing arteriopathies and precipitating stressors and cardiovascular outcomes. Circ Cardiovasc Interv 2014; 7: 645-655.

8. Eleid MF, Guddeti R, Tweet MS, Lerman A, Singh M, Best PJ, et al. Coronary artery tortuosity in spontaneous coronary artery dissection: Angiographic characteristics and clinical implications. Circ Cardiovasc Interv 2014; 7: 656-662.

9. Tweet MS, Eleid MF, Best PJ, Lennon RJ, Lerman A, Rihal CS, et al. Spontaneous coronary artery dissection: Revascularization versus conservative therapy. Circ Cardiovasc Interv 2014; 7: $777-786$.

10. Rogowski S, Maeder MT, Weilenmann D, Haager PK, Ammann $\mathrm{P}$, Rohner F, et al. Spontaneous coronary artery dissection: Angiographic follow-up and long-term clinical outcome in a predominantly medically treated population. Catheter Cardiovasc Interv 2017; 89: 59-68.

11. Nakashima T, Noguchi T, Haruta S, Yamamoto Y, Oshima S, Nakao K, et al. Prognostic impact of spontaneous coronary artery dissection in young female patients with acute myocardial infarction: A report from the Angina Pectoris-Myocardial Infarction Multicenter Investigators in Japan. Int J Cardiol 2016;
207: $341-348$.

12. Saw J. Spontaneous coronary artery dissection. Can J Cardiol 2013; 29: $1027-1033$.

13. Eleid MF, Tweet MS, Young PM, Williamson E, Hayes SN, Gulati R. Spontaneous coronary artery dissection: Challenges of coronary computed tomography angiography. Eur Heart J Acute Cardiovasc Care 2018; 7: 609-613.

14. Alzand BS, Vanneste L, Fonck D, Van Mieghem C. Spontaneous coronary artery dissection undissolved using cardiac computed tomography. Int J Cardiol 2016; 222: 1040-1041.

15. Rashid HN, Wong DT, Wijesekera H, Gutman SJ, Shanmugam VB, Gulati R, et al. Incidence and characterisation of spontaneous coronary artery dissection as a cause of acute coronary syndrome: A single-centre Australian experience. Int J Cardiol 2016; 202: $336-338$

16. Hassan S, Prakash R, Starovoytov A, Saw J. Natural history of spontaneous coronary artery dissection with spontaneous angiographic healing. JACC Cardiovasc Interv 2019; 12: 518-527.

17. Saw J, Humphries K, Aymong E, Sedlak T, Prakash R, Starovoytov A, et al. Spontaneous coronary artery dissection: Clinical outcomes and risk of recurrence. Am J Coll Cardiol 2017; 70: $1148-1158$ 